\title{
Breathing kagome XY quantum magnet with four-site ring exchange
}

\author{
Niklas Casper ${ }^{*}$ and Wolfram Brenig ${ }^{\dagger}$ \\ Institute for Theoretical Physics, Technical University Braunschweig, D-38106 Braunschweig, Germany
}

(Dated: February 22, 2021)

\begin{abstract}
We study the impact of trimerization (breathing) of the nearest-neighbor (NN) exchange on the planar XY spin-1/2 ferromagnet on the kagome lattice, including additional four-site ring exchange. For uniform NN exchange, this model has previously been shown to transit from a long-range ordered ferromagnet into a $\mathbb{Z}_{2}$ quantum spin liquid by virtue of the ring exchange. Using quantum MonteCarlo calculations, based on the stochastic series expansion, we present results for the spin stiffness, the quantum phase diagram, the longitudinal static, as well as the transverse dynamic structure factor. Our results corroborate 3D XY universality also at finite trimerization and suggest a simple continuation of the quantum critical point of the uniform case into a line in terms of rescaled exchange parameters. Moreover, at any trimerization and in the ordered phase the elementary excitations can be understood very well in terms of linear spin wave theory, while beyond the critical line, in the spin liquid phase we find signatures of spinon continua.
\end{abstract}

\section{INTRODUCTION}

Quantum spin liquids (QSL) constitute intriguing forms of magnetic matter, which are attracting great interest since several decades by now [1 6]. QSLs are characterized by the absence of local magnetic order parameters, even at zero temperature, they show fractionalized excitations, long-ranged topological entanglement, and quantum orders beyond Landau's paradigm of symmetries and spontaneous symmetry breaking. Proposals for putative QSLs mostly rest on an underlying gauge structure [7, e.g. $U(1)$ or $\mathbb{Z}_{2}$, likely related to the non-locality of the fractional excitations [5].

Frustration of spin interactions is a prime ingredient to drive magnetic systems into QSL states. This renders quantum Monte-Carlo (QMC) calculations widely inapplicable as an unbiased tool, because of the minus-sign problem [8]. Among the few exceptions, in which a $\mathbb{Z}_{2}$ QSLs can exactly be shown to exists in $D \geq 2$ dimensions [9] and for which quantum Monte-Carlo methods can be applied [10, is Kitaev's spin model, with frustrated compass exchange on the honeycomb lattice. Another case of great interest are variants of the class of Balents-FisherGirvin (BFG) type models [1], which comprise certain XXZ Hamiltonians with Ising frustration and easy-axis anisotropy, e.g. on the kagome lattice. In the stronganisotropy limit, the low-energy manifolds of these models are exponentially degenerate from $S^{z}$ constraints on local units and feature ring exchange $K \sim J_{X Y}^{2} / J_{z}$. The latter can lead to dynamics dual to quantum dimer models [12, 13] and realizes a similar $\mathbb{Z}_{2}$ gauge structure [1420.

Consequently the BFG QSLs are topologically ordered with four-fold ground state degeneracy on the torus in 2D and represent a deconfined phase [21 23]. The deconfined non-local elementary excitations are analogous to

\footnotetext{
* n.casper@tu-bs.de

$\dagger$ w.brenig@tu-bs.de
}

those of the toric code, i.e. 'electric' e-particles (spinons, neutral spin- $1 / 2$ excitations) and 'magnetic' $m$-particles (vortices, visons) [5, 13, 23, 24]. The $e$ - and $m$-particles are relative semions [5, 23], and in the BFG models the spinons are known to have bosonic statistics [11. As for additional fingerprints of a $\mathbb{Z}_{2}$ QSL, the BFG phase hosts symmetry-protected edge states for open boundary conditions [25] and displays topological entanglement entropy [19, 20].

An explicit low-energy Hamiltonian to study the BFG QSL on the kagome lattice is the XY-model with ring exchange on four-site bow-ties [18, 26], as in Fig. 1 ]

$$
H=-\frac{1}{2} \sum_{\langle i j\rangle} J_{i j}\left(S_{i}^{+} S_{j}^{-}+\text {h.c. }\right)-K \sum_{\langle i j k l\rangle \in \bowtie} P_{i j k l},
$$

where $S_{i}^{ \pm}$are spin-1/2 raising and lowering operators on sites $i$ and $J_{i j} \geq 0$ and $K \geq 0$ are the nearest-neighbor(NN) and ring exchange constants, respectively. The ring exchange $P_{i j k l}=S_{i}^{+} S_{j}^{-} S_{k}^{+} S_{l}^{-}+S_{i}^{-} S_{j}^{+} S_{k}^{-} S_{l}^{+}$acts on each bow-tie $(\bowtie)$ as illustrated in Fig. 11(a). This model is amenable to QMC analysis, as it lacks a minus-sign problem. Variants of it, introducing additional interactions, including also the $S^{z}$ components have been considered, focusing either on the spin, or its equivalent hard-core boson formulation [15, 17, 19, 20, 27].

With nearest-neighbor exchange $J_{i j}=J$, Hamiltonian (1) has been shown to harbor two quantum phases versus $J / K[18$. For $K \rightarrow 0$ the model represents the spin-1/2 XY ferromagnet (FM), displaying a superfluid (SFL) phase in terms of the hard-core boson language. For $J \rightarrow 0$ a BFG QSL is established. The critical coupling is $(K / J)_{c} \approx 10.9$ [18, 59]. Approaching the critical point out of the QSL, the spinons condense into the superfluid density $\langle b\rangle$ of the hard-core bosons, undergoing a conventional XY transition. The latter features standard values for the exponent $\nu \simeq 0.6717$ of the divergence of the correlation length and $z=1$ for the dynamical critical exponent, consistent with 3D XY universality.

Remarkably however, due to the composite nature of the hard-core bosons in terms of the spinons, the expo- 
nent of the equal-time boson-correlation function turns into a fingerprint of the deconfined quantum critical nature of the transition 28 . In fact, $\eta^{\star} \approx 1.339-1.493$ has been established [20, 28, 33, which is different from standard 3D XY universality, i.e. $\eta \approx 0.038$ [34], and therefore is referred to as $\mathrm{XY}^{\star}$.

A natural extension of (1) is to include inhomogeneity of the NN exchange in terms of trimerization, also known as breathing, in which the spins belonging to upward(downward) facing triangles experience different exchange couplings, $J_{\Delta(\nabla)}$. Such generalization is motivated both by theory and experiment. With respect to the former, recent analysis of other breathing kagome spin systems, i.e. antiferromagnetic XYZ models indicate that trimerization may help to stabilize quantum disordered phases and QSLs [35 41]. However for BFGtype models such analysis is lacking. With respect to experiment, several interacting hard-core boson systems on breathing kagome lattices have been realized in recent ultracold-atom systems [42,46].

In this context, the main purpose of this work is to uncover the quantum magnetism of the XY model with ring exchange on the breathing kagome lattice versus the trimerization ratio. Our prime focus will be on the spin stiffness and the static spin structure factor (SSSF) in order to analyze the shift of the quantum critical point (QCP) versus $J_{\Delta} / J_{\nabla}$. In addition we will consider the dynamical spin structure factor (DSSF) in order to shed light on the elementary excitations.

The outline of the paper is as follows. In Section II A, we list several details of the model. Section II B sketches the QMC method. In Section II C, the extraction from QMC of observables relevant to our study is described. Section III is devoted to our results, comprising the spin stiffness in Section III A, the quantum phase diagram in Section III B the static and dynamic structure factors in Sections IIIC and IIID. We conclude in Section IV. We provide Appendix $\mathrm{A}$ on the single trimer stiffness and Appendix $B$ on the linear spin wave theory (LSWT) for the breathing XY kagome ferromagnet.

\section{MODEL AND METHOD}

\section{A. Model}

The breathing version of model (1) reads

$$
\begin{aligned}
H= & -\frac{1}{2}\left(\sum_{\Delta\langle i j\rangle} J_{\Delta} S_{i}^{+} S_{j}^{-}+\sum_{\nabla\langle i j\rangle} J_{\nabla} S_{i}^{+} S_{j}^{-}+\text {h.c. }\right) \\
& -K \sum_{\langle i j k l\rangle \in \bowtie} P_{i j k l},
\end{aligned}
$$

where $J_{\Delta(\nabla)}$ refer to the solid(dashed) up(down) triangles on the kagome lattice, depicted in Fig. 11(a), which is a triangular Bravais lattice with lattice vectors $\mathbf{R}_{1,2}=\left(\frac{1}{2}, \frac{\sqrt{3}}{2}\right),(1,0)$ for a lattice constant $l=1$ hereafter,
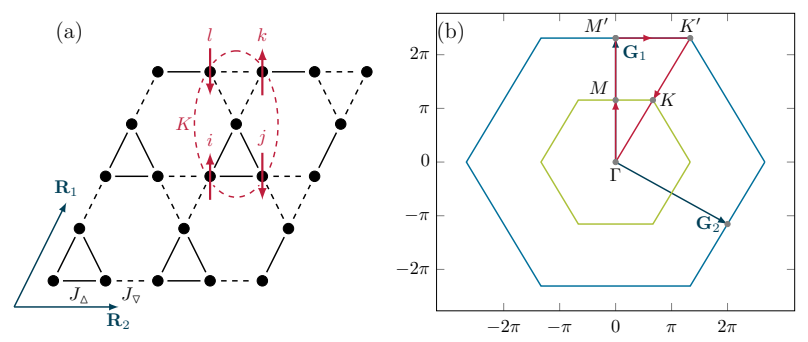

FIG. 1. (a) Trimerized kagome lattice, with NN and ring exchange $J_{\Delta(\nabla)}$ and $K$. (b) First and second Brillouin zone with high-symmetry path.

and a three-site basis at $\mathbf{r}_{\alpha=0,1,2}=(0,0),\left(\frac{1}{4}, \frac{\sqrt{3}}{4}\right),\left(\frac{1}{2}, 0\right)$, i.e. the intersite distance is $a=1 / 2$ and the number of spins is $N=3 L^{2}$. The reciprocal lattice vectors are $\mathbf{G}_{1,2}=\left(0, \frac{4 \pi}{\sqrt{3}}\right),\left(2 \pi,-\frac{2 \pi}{\sqrt{3}}\right)$, with $\mathbf{G}_{i} \cdot \mathbf{R}_{j}=2 \pi \delta_{i j}$ and the Brillouin zone (BZ) is set by $\mathbf{q}=\frac{n_{1}}{L} \mathbf{G}_{1}+\frac{n_{2}}{L} \mathbf{G}_{2}$ with $n_{1,2}=0,1, \ldots, L-1$, see Fig. 1(b).

For the remainder of the text we will employ a number of dimensionless parameters, i.e. $j=J_{\nabla} / J_{\Delta}, k=K / J_{\Delta}$ and $\kappa=K / \bar{J}$. There we define a mean exchange coupling $\bar{J}=\left(J_{\Delta}+J_{\nabla}\right) / 2$ with a dimensionless version of $\bar{\jmath}=\bar{J} / J_{\Delta}=(1+j) / 2$. In this work, we will consider the region of $0 \leq j \leq 1$ and $k \geq 0$.

\section{B. Quantum Monte-Carlo method}

The numerical results of this work are obtained from QMC calculations, using the stochastic series expansion (SSE) 47 49. This method is based on an importance sampling of the high temperature series expansion of the partition function

$$
\mathcal{Z}=\sum_{\alpha} \sum_{S_{M}} \frac{(-\beta)^{n}(M-n) !}{M !}\left\langle\alpha\left|\prod_{a=1}^{M} H_{t_{a}, p_{a}}\right| \alpha\right\rangle
$$

where $\beta=1 / T$ is the inverse temperature and $M$ the truncation order, self-adjusted to the desired precision. As compared to conventional implementations, we enhance the approach by including the ring exchange following Ref. [50]. I.e. the entries $H_{t_{a}, p_{a}}$ of the operator string $\prod_{a=1}^{M} H_{t_{a}, p_{a}}$ now comprise $C-, J_{-}$, and $K$-type operators at plaquette $p, H_{C, p}=C I_{i j k l}, H_{J, p}=\left(S_{i}^{+} S_{j}^{-}+S_{i}^{-} S_{j}^{+}\right) I_{k l} / 2$, and $H_{K, p}=S_{i}^{+} S_{j}^{-} S_{k}^{+} S_{l}^{-}+S_{i}^{-} S_{j}^{+} S_{k}^{-} S_{l}^{+}$, including appropriate permutations of ijkl. $C$ has to be chosen such that all weights of the $C$-type operators are nonnegative. $|\alpha\rangle=\left|S_{1}^{z}, \ldots, S_{N}^{z}\right\rangle$ refers to the $S^{z}$ basis and $S_{M}=\left[t_{1}, p_{1}\right]\left[t_{2}, p_{2}\right] \ldots\left[t_{M}, p_{M}\right]$ is an index for the operator string.

This operator string is sampled, using a Markovianchain Metropolis scheme, employing three types of updates, i.e. (i) diagonal updates which change the number of $C$-type operators $H_{C, p}$ in the operator string, (ii) loop 
updates which change the type of operators $H_{C, p} \leftrightarrow H_{J, p}$ and $H_{J, p} \leftrightarrow H_{K, p}$, and (iii) multibranch cluster update which change the type of operators $H_{C, p} \leftrightarrow H_{K, p}$. The latter update refers to the prime new ingredient for ring exchange models [50. For bipartite lattices the loop update comprises an even number of off-diagonal operators ( $J$ - and $K$-type). This ensures positivity of the transition probabilities and waives the minus-sign problem.

\section{Observables}

Here we briefly sketch formal details regarding the main physical observables which we evaluate.

\section{Spin Stiffness}

To obtain the superfluid density, we calculate the spin stiffness (or helicity modulus) $\rho_{S}$

$$
\rho_{S}=\left.\frac{\partial^{2} F(\phi)}{\partial \phi^{2}}\right|_{\phi=0}
$$

which indicates the presence of long-range order (LRO). Here $F(\phi)$ is the free energy versus a twist of the spins in the XY-plane, with an angle increasing by $\phi=\Phi / L$ per bond for any given bond direction. At $T=0$, the free energy is replaced by the ground state energy $E(\phi)$. We implement the estimator for this quantity following Refs. 51 54, using that the r.h.s. of (4) can be expressed in term of squares of operators $S_{i}^{+} S_{j}^{=}$, transporting $\uparrow$-spins along the $i j$-bond. I.e.

$$
\rho_{S}=\frac{1}{d \beta} \sum_{\alpha}^{d}\left\langle W_{\alpha}^{2}\right\rangle
$$

where $d=2$ refers to the dimension and the winding number $W_{\alpha}$ is defined by

$$
W_{\alpha}=\frac{1}{L_{\alpha}} \sum_{b} N_{b, \alpha}
$$

where $\alpha=x, y$ is the spatial direction and $L_{\alpha}$ the number of bonds per spatial direction. The sign of the phase factor is $N_{b, \alpha}= \pm 1$ whether the bond operator transports a net spin current in + or - spatial direction, which corresponds to the operators $S^{+} S^{-}$or $S^{-} S^{+}$in the operator string.

\section{Spin Structure Factors}

We evaluate two types of spin correlation functions. First, the longitudinal, i.e $z z$, static spin structure factor (SSSF) [18]

$$
S(\mathbf{q})=\frac{1}{N} \sum_{i \alpha, j \beta} e^{\mathrm{i} \mathbf{q} \cdot\left(\mathbf{R}_{i \alpha}-\mathbf{R}_{j \beta}\right)}\left\langle S_{i \alpha}^{z} S_{j \beta}^{z}\right\rangle,
$$

where $\left\langle S_{i \alpha}^{z} S_{j \beta}^{z}\right\rangle$ is extracted during the simulation, and the sites $i \alpha$ correspond to lattice coordinates $\mathbf{R}_{i \alpha}=n_{1 i} \mathbf{R}_{1}+n_{2 i} \mathbf{R}_{2}+\mathbf{r}_{\alpha}$, with $n_{1 i(2 i)}=0,1, \ldots, L-1$.

Additionally we evaluate the transverse, i.e. +-, dynamic spin structure factor (DSSF). In real space and at imaginary time $\tau$ this is obtained from the SSE by [4]

$$
\begin{aligned}
&\left\langle S_{i}^{+}(\tau) S_{j}^{-}(0)\right\rangle=\left\langle\sum_{m=0}^{M}\left(\begin{array}{c}
M \\
m
\end{array}\right)\left(\frac{\tau}{\beta}\right)^{m}\left(1-\frac{\tau}{\beta}\right)^{M-m}\right. \\
&\left.\frac{1}{M} \sum_{p=0}^{M-1} S_{i}^{+}(m+p) S_{j}^{-}(p)\right\rangle_{W}
\end{aligned}
$$

where $i, j$ refer to any lattice site, $m+p, p$ label positions within the operator string, i.e. intermediate time slices, and $\langle\ldots\rangle_{W}$ denotes the Metropolis weight of an operator string of length $M$ generated by the SSE [48, 49].

From (8) we proceed to momentum space by Fourier transformation and define

$$
S_{\alpha \beta}(\mathbf{q}, \tau)=\frac{1}{N} \sum_{i, j} e^{\mathrm{i} \mathbf{q} \cdot\left(\mathbf{R}_{i \alpha}-\mathbf{R}_{j \beta}\right)}\left\langle S_{i \alpha}^{+}(\tau) S_{j \beta}^{-}(0)\right\rangle
$$

which due to the three-sites basis is a $3 \times 3$ matrix. To analyze the excitation spectrum, we follow Ref. [26] and confine ourselves to the analytic continuation of the trace $\sum_{\alpha} S_{\alpha, \alpha}(\mathbf{q}, \tau)$. This trace is invariant with respect to any unitary transformation of $S_{\alpha, \beta}(\mathbf{q}, \tau)$ and in particular we may rotate onto its principal axis. I.e. we diagonalize this matrix for each $\mathbf{q}$ and $\tau$ and transform to real frequencies $\omega$ for each of the eigenvalue $S_{\mu}(\mathbf{q}, \tau), \mu=0,1,2$ separately

$$
S_{\mu}(\mathbf{q}, \tau)=\int_{0}^{\infty} d \omega S_{\mu}(\mathbf{q}, \omega) K(\omega, \tau)
$$

with a kernel $K(\omega, \tau)=\left(e^{-\tau \omega}+e^{-(\beta-\tau) \omega}\right) / \pi$. We find, the eigenvalue decomposition to allow for a higher resolution in the analytic continuation, if regions of dominant spectral features occur nearby in energy space. In principle, only $\sum_{\mu} S_{\mu}(\mathbf{q}, \omega)$ refers to the actual total spectrum and the $S_{\mu}(\mathbf{q}, \omega)$ are auxiliary functions, which do not necessarily encode any decomposition into sharp eigenmodes. Yet, visualizing each $S_{\mu}(\mathbf{q}, \omega)$ can be instructive.

The inversion 10 is an ill-posed problem, for which maximum entropy methods (MEM) have proven to be well suited. Here we use Bryan's MEM algorithm 55 57. This method minimizes the functional $Q=\chi^{2} / 2-\alpha \sigma$, with $\chi$ being the covariance of the QMC data with respect to the MEM trial spectrum $S_{\mu}(\mathbf{q}, \omega)$. Overfitting is prevented by an entropy term $\sigma=\sum_{\omega} S_{\mu}(\mathbf{q}, \omega) \ln \left[S_{\mu}(\mathbf{q}, \omega) / m(\omega)\right]$. We have used a flat default model $m(\omega)$, which is iteratively adjusted to match the zeroth moment of the trial spectrum. The optimal spectrum follows from the average of $S_{\mu}(\mathbf{q}, \omega)$, weighted by a probability distribution $P\left[\alpha \mid S_{\mu}(\mathbf{q}, \omega)\right][55]$. 


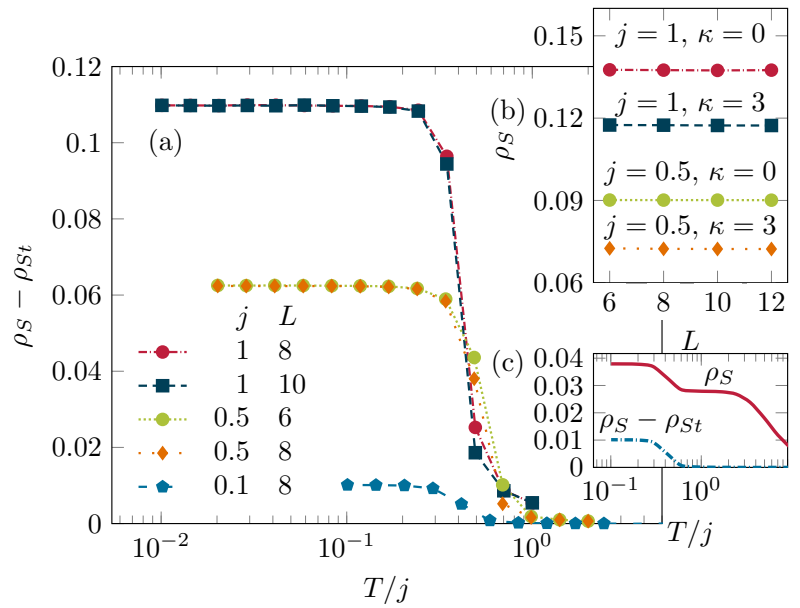

FIG. 2. (a) Spin stiffness $\rho_{S}$ for $\kappa=0$, subtracted by single trimer spin stiffness $\rho_{S t}$, for various trimerization ratios $j=1,0.5,0.1$ shown over wide temperature range. Lowtemperature plateaus set the scale $T^{*}=j / L$. Note: $x$-axis scaled by $j$. (b) Inset shows spin stiffness versus $L=6, \ldots, 12$ at fixed low temperature $T=j / 10$, for various trimerization ratios $j=1,0.5$ and ring exchange $\kappa=0,3$. Errorbars smaller than marker size. (c) Inset shows spin stiffness $\rho_{S}$ and $\rho_{S}-\rho_{S t}$ at $L=8$ and $j=0.1$ versus temperature.

\section{RESULTS}

In this section, we detail our findings for the spin stiffness and the quantum phase diagram, as well as the static, and dynamic structure factors in order to characterize the phases of the model versus ring exchange and trimerization.

\section{A. Spin Stiffness}

We use the stiffness to extract the quantum critical point. To this end we rely on the fact that scaling theory [58] has been proven to apply for $j=1$ [18] and we anticipate that chosing $j \neq 1$ should not change this. This implies that the stiffness fulfills the scaling relation $\rho_{S}=L^{-z} F\left(\left(\kappa_{C}-\kappa\right) L^{1 / \nu}, \beta / L^{z}\right)$ with a universal scaling function $F$. In turn, using $z=1$ for the dynamical critical exponent, and fixing the temperature such that $L^{z} / \beta=c$ is constant, i.e. $T=T^{\star}=c / L$, the function $\rho_{S} L$ will collapse onto a single curve versus $\left(\kappa_{C}-\kappa\right) L^{1 / \nu}$ for all $L$. We use a small constant $c$, such that $\rho_{S}$ represent the zero temperature limit for $T<T^{\star}$ for each $L$ studied. Furthermore, since $\rho_{S}(\kappa=$ const., $L)=$ const. for $L \gg 1$ and $T \rightarrow 0$, this implies that $F(x$, const $) \propto x^{\nu}$.

In Fig. 2(a), and prior to analyzing the scaling behavior, we display the spin stiffness versus temperature for various trimerization at $\kappa=0$ where LRO is certain at $T=0$ for $j=1$. For $j \rightarrow 0$, a stiffness $\rho_{S t}$ of isolated trimers remains, which we subtract off for simplicity. The impact of retaining $\rho_{S t}$ is exemplified for

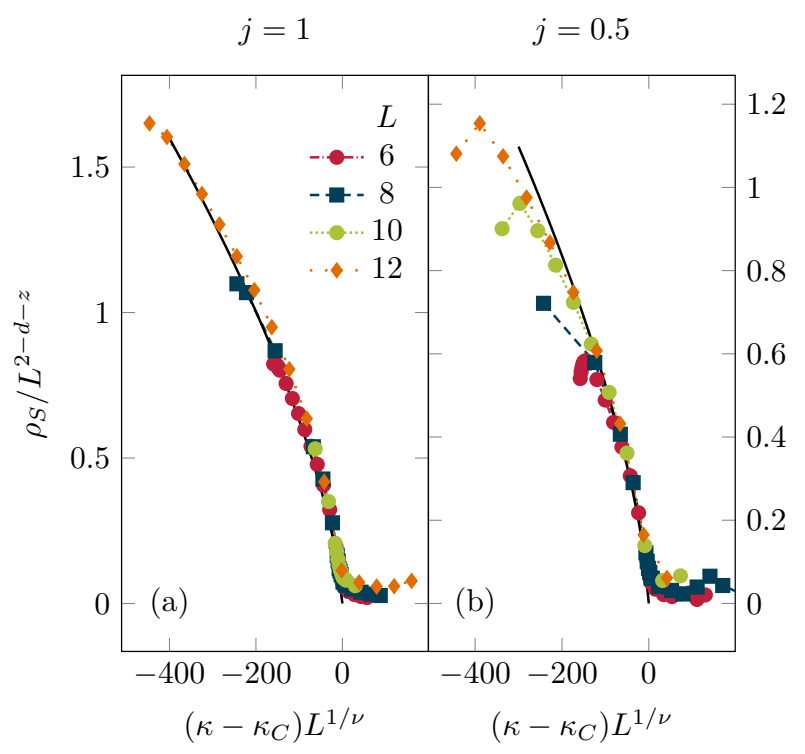

FIG. 3. Scaling behavior at $T^{*}=j / L$ of $\rho_{S} / L^{2-d-z}$ versus $\left(\kappa-\kappa_{C}\right) L^{1 / \nu}$ at (a) $j=1$ and (b) $j=0.5$. For $j=1(j=0.5)$ QMC data collapses at $\kappa_{C} \approx 11.04\left(\kappa_{C} \approx 10.97\right)$. Solid black curve $\propto\left(\kappa_{C}-\kappa\right)^{\nu}$.

$j=0.1$ in Fig. 2(c). Several points can be read off from this figure. First, there is a clear low- $T$ crossover regime to a state with a finite stiffness, which decreases with increasing trimerization. Choosing $T^{\star}$ well below this regime is sufficient for the scaling analysis to describe zero temperature behavior. Second, the crossover regimes for different $j$ can be made to coincide, if $T$ is scaled by $j$. Third, the figure shows that the crossover regime is rather insensitive with respect to $L$ for the systems sizes we have investigated. Therefore, we use $c \simeq j$, i.e. $T^{\star}=j / L$ as a reasonable choice to obtain quasi ground state properties.

We also note from Fig. 2(a), that the low- $T$ saturation value of $\rho_{S}-\rho_{S t}$ depends little on system size. Sufficiently close to criticality, this is to be expected from scaling. I.e., for any temperature on the low- $T$ saturation plateau of $\rho_{S(t)}$, the universal function $F(x, y)$ is in its asymptotic regime $F(x$, const $) \propto x^{\nu}$. At fixed $\kappa_{(C)}$ therefore, $\rho_{S} \propto L^{-z}\left(L^{1 / \nu}\right)^{\nu}=$ const versus $L$. For $\rho_{S t}$ the latter is satisfied trivially. The inset Fig. 2(b) demonstrates, that the independence of system size of $\rho_{S}$ at low $T$ remains valid over a wide range of $j$ - and $\kappa$-values, irrespective of criticality, rendering the usage of the scaling function rather robust.

Setting $T=T^{\star}$, we now extract the QCP by optimizing the collapse of all of our results for $\rho_{S} L^{z}$ versus $\left(\kappa_{C}-\kappa\right) L^{1 / \nu}$ for $L=6 \ldots 12$ at fixed $j$, for various $j=0.1 \ldots 1$ and employing the $3 \mathrm{D} \mathrm{XY}^{\star}$ universality established, i.e. $z=1$ and $\nu=0.6717$. This leaves $\kappa_{C}$ as the single fit parameter.

Results for this optimization procedures are depicted in Fig. 3 for two different $j$. Similar behavior is obtained 


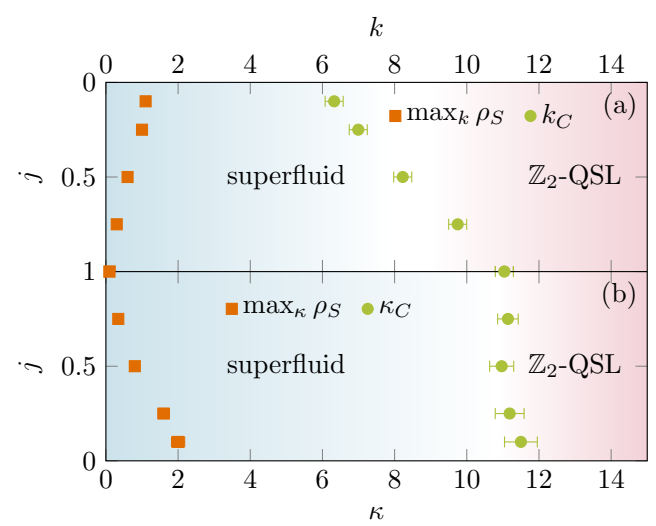

FIG. 4. Quantum phase diagram versus (a) ring exchange $k$, (b) dimensionless ring exchange $\kappa$ and trimerization ratio $j$. Green symbols, critical coupling (a) $k_{C}$, (b) $\kappa_{C}$ for SFLQSL QCP. Orange squares, location of $\rho_{S}$-maximum. If not visible, errrobars smaller than marker size. Note: opposite $y$-axis directions in (a) and (b).

for all other $j$ considered. The collapse is clearly evident and leads to a critical ring exchange $\kappa_{C} \approx 11.04$ and 10.97 at $j=1$ and 0.5 , respectively. The value for $\kappa_{C}(j=1)$ agrees with previous findings [18. The figure displays an additional function $\propto\left(\kappa-\kappa_{C}\right)^{\nu}$, which can be superimposed to fit the QMC results very well in the ordered phase. Apart from the collapse itself, this provides further support for the validity of the scaling hypothesis also at finite trimerization. In the LRO phase at $j \neq 1$, and significantly off from criticality, non-universal corrections in terms of an intermediate maximum in $\rho_{S} L$ appear at finite $\kappa$. We observe this maximum at all $j \neq 1$ investigated. Yet, since $\rho_{S}$ remains finite, the stiffness provides no evidence for phases other than the SFL and QSL at $j \neq 1$.

\section{B. Quantum Phase Diagram}

Using all $\kappa_{C}(j)$ obtained by the data collapse, we are in a position to extend the quantum phase diagram (QPD) derived in Ref. [18 from the line $j=1$ onto the $(\kappa, j)$ plane. This is shown in Fig. 4(b). Remarkably, if expressed in terms of $\kappa=2 K /\left(J_{\Delta}+J_{\nabla}\right)=2\left(K / J_{\Delta}\right) /(1+j)$ the transition resides at a fixed location versus $j$ within the error of the data collapse. Speaking differently, and equally remarkable, removing the implicit $j$-dependence from the $x$-axis, as in Fig. 4(a), the QPD reveals an increase in the tendency to form the QSL as the trimerization increases. This can be understood by the decrease with $j$ of the boson kinetic energy which drives the SFL phase. In fact, performing linear spin-wave theory (LSWT) exactly at $K=0$, see Appendix B, each spin is connected to four neighboring ones, two of them by $J_{\Delta}$ and two of them by $J_{\nabla}$. Therefore the leading order-1/S contribution to the energy is proportional to

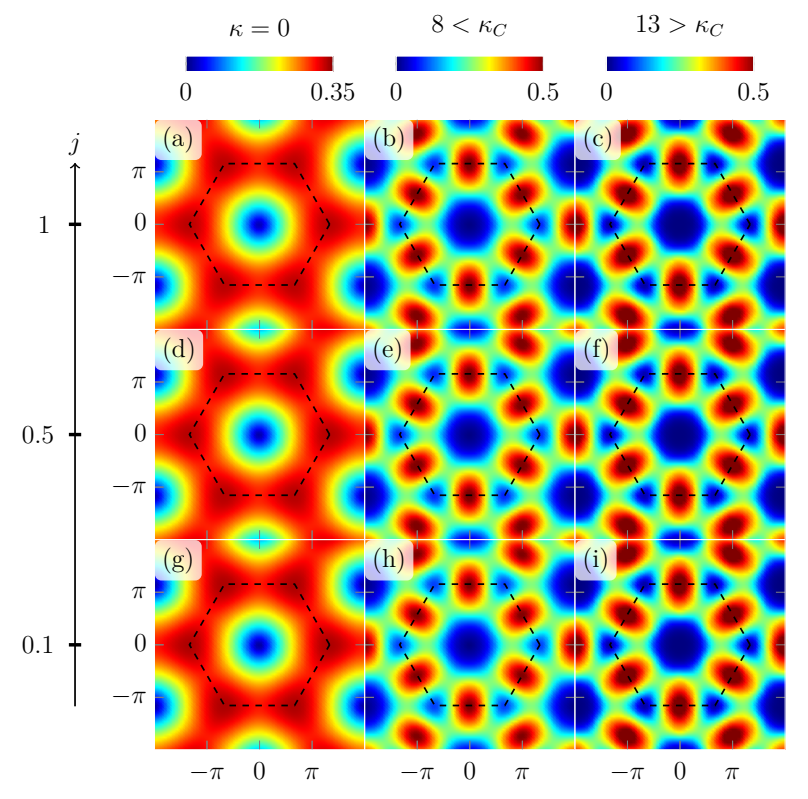

FIG. 5. SSSF $S(\mathbf{q})$ for various trimerization ratios $j=1,0.5,0.1$ and ring exchange couplings $\kappa=0,8\left(<\kappa_{C}\right)$, and $13\left(>\kappa_{C}\right)$. QMC at $L=12, T=T^{*}$.

$\bar{\jmath}$, see Eqns. (B4, B5).

It is tempting to speculate about the QPD as $j \rightarrow 0$. First, convergence issues with the QMC prevent us from studying $j=0$ directly. Second, strictly at $j=\kappa=0$, there can be no SFL LRO phase, since the system is a lattice of disconnected trimers. Finally, it is likely, that the QSL remains existent for $\kappa>\kappa_{C}$, also at $j=0$. For $\kappa<\kappa_{C}$, one scenario could be that the superfluid density vanishes as $j \rightarrow 0$, consistent with Fig. 2, such that $\rho_{S} \leq \rho_{S t}$ on the line $j=0$ for all $\kappa<\kappa_{C}$. However, this renders the nature of the state for $\kappa<\kappa_{C}$ at $j=0$ unclear. Another scenario, suggested by the maximum in $\rho_{S}$ could be, that weak LRO, driven by ring exchange, remains present even at $j=0$, but with a non-monotonous behavior versus $\kappa<\kappa_{C}$. This remains an open issue.

\section{Static Spin Structure Factor}

The $\operatorname{SSSF} S(\mathbf{q})$ is equivalent to the static densitydensity correlation function within the hard-core boson picture. As such it has been used to check if the transition from the SFL into the anticipated $\mathbb{Z}_{2}$-QSL is accompanied by a density wave, i.e. by any peaks scaling $\propto N$. This has been excluded in [18] at $j=1$, proving that the non-SFL phase does not break discrete lattice symmetries and thereby further corroborating its QSL nature.

In view of the QPD in Fig. 4, the short-range nature of the $z z$-spin-correlations will remain true also for $j<1$. Therefore, rather than repeating the finite-size scaling analysis of 18, we highlight instead, that the SSSF is almost insensitive to trimerization if considered in terms 

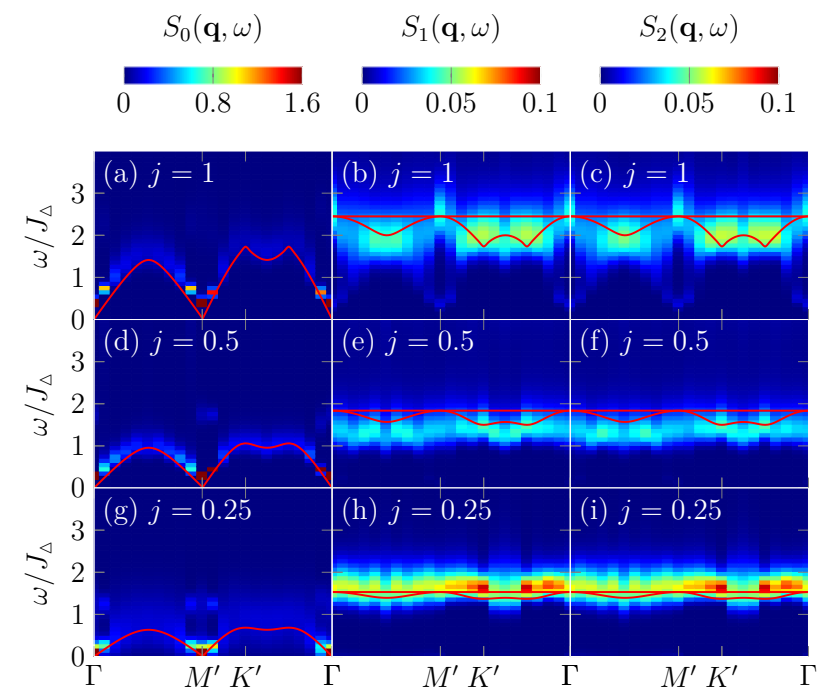

FIG. 6. DSSF in SFL phase at $\kappa=0$ for three trimerization ratios $j=1,0.5,0.25$ (rows), along high-symmetry path Fig. 1 (b) in first and second BZ. Columns refer to the three diagonal modes $S_{\mu}(\mathbf{q}, \omega)$ in Eq. 10. $\sum_{\mu} S_{\mu}(\mathbf{q}, \omega)$ yields total spectrum. QMC at $L=12, T=T^{*}$. Solid red line: LSWT dispersions Eqns. B4 B5.

of the rescaled ring exchange $\kappa$. This is shown in Fig. 5 , which depicts contours of $S(\mathbf{q})$ at $T=T^{*}$ and for $L=12$ at $\kappa=0,8\left(<\kappa_{C}\right)$, and $13\left(>\kappa_{C}\right)$.

Indeed, the changes in this figure along the vertical $j$ direction in each column are small only. The evolution of the contours along the $\kappa$-direction within the $j=1$ row are consistent with that in [18]. For $\kappa \ll 1$, the maximum intensity in $S(\mathbf{q})$ resides at the $\mathbf{K}$-points, due to the NN-correlations from $j$. As $\kappa$ increases, the next NN-correlations produce maximum intensity at the $\mathbf{M}$ points and minimum intensity at the $\boldsymbol{\Gamma}$ - and $\mathbf{K}$-points.

\section{Dynamic Spin Structure Factor}

As to be expected from the QPD, the elementary excitations fall into one of two classes. I.e. for $\kappa<\kappa_{C}$ they are magnons of a planar FM, with a broken residual $U(1)$ symmetry and for $\kappa>\kappa_{C}$ they represent the deconfined spinons and visons of the $\mathbb{Z}_{2}$-QSL. While the DSSF can be used directly to map out the magnon spectrum, it cannot do so for the fractionalized one-particle excitations. However, since the DSSF comprises a two-particle correlation function in terms of the latter, it can access the two-spinon spectrum, which is a continuum at each fixed total momentum. Using QMC at $j=1$, magnons (twospinon continua) have been verified in the FM (QSL) phase directly for Hamiltonian (2) 27, and for a closely related model of the BFG class 26. Here we consider the DSSF for $j \leq 1$. Primarily, we focus on the magnon excitations at $\kappa=0$, since the dynamics in the QSL phase is driven by the ring exchange, where effects of the

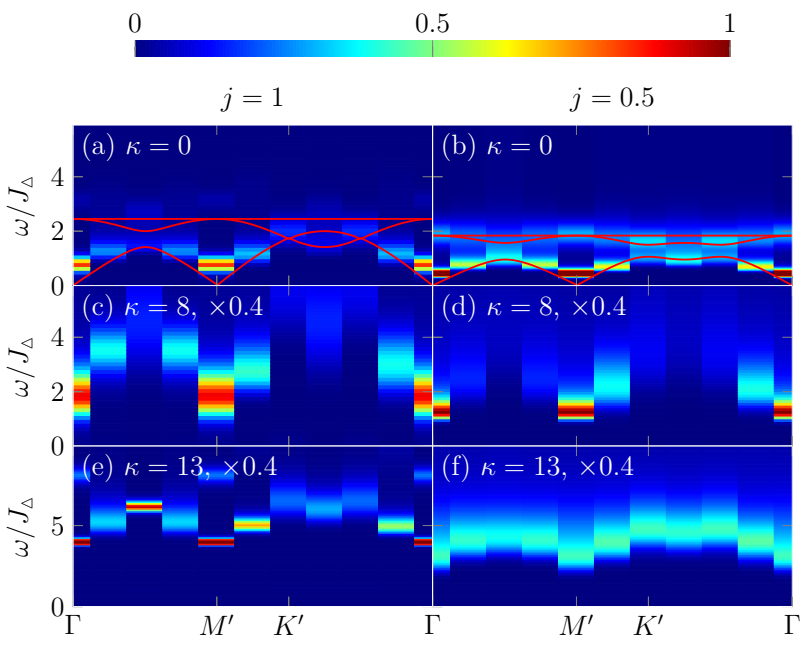

FIG. 7. DSSF total spectrum $\sum_{\mu} S_{\mu}(\mathbf{q}, \omega)$ for three ring exchange values, in SFL phase at $\kappa=0,8$ (first, second row), and QSL phase at $\kappa=13$ (third row) for two trimerization ratios $j=1,0.5$, along high-symmetry path Fig. 1(b) in first and second BZ. QMC at $L=6$ and $T=T^{*}$. Intensities rescaled for visibility. Solid red line in first row: LSWT dispersions Eqns. B4 B5.

trimerization are not expected to be significant.

Fig. 6 details the evolution with $j$ of the spectra of the three auxiliary diagonal modes $S_{\mu}(\mathbf{q}, \omega)$ of the DSSF at $\kappa=0$, with the total spectrum being $\sum_{\mu} S_{\mu}(\mathbf{q}, \omega)$. The dispersions are shown along the high symmetry path of the extended BZ depicted in Fig. 1(b). Each rows of the figure display the typical signatures of a FM with a three-site unit cell. I.e. there is one "acoustic" Goldstone magnon and two "optical" magnon branches. Properly, the dominant spectral weight occurs in the Goldstone mode at the ordering vector, i.e. the $\boldsymbol{\Gamma}$-point. The effect of the trimerization can be seen clearly along the columns of Fig. 6, i.e. as $j$ is decreased, implying a reduction of the magnon kinectic energy, the bandwidth for both types of magnons shrinks accordingly, with however the Goldstone nature of the acoustic mode remaining intact.

To obtain a qualitative account of the magnon energies to leading $O(1 / S)$ we have performed LSWT. See Appendix B. The LSWT dispersions are included in Fig. 6. Regarding the Goldstone mode, the LSWT dispersion agrees remarkably well with $S_{0}(\mathbf{q}, \omega)$ from the QMC. For the two optical modes of the LSWT, $S_{1}(\mathbf{q}, \omega)$ and $S_{2}(\mathbf{q}, \omega)$ certainly are within their energy range, however they do not separate into distinct branches. Irrespective of the qualitative agreement between QMC and LSWT, the figure also clearly demonstrates, that at higher energies the QMC spectra tend to broaden, hinting at the relevance of magnon-magnon interactions.

Fig. 7 depicts the evolution of the DSSF with $\kappa$ crossing over from the SFL into the QSL phase, along two lines with $j=1$ and 0.5 . Each spectrum refers directly to the trace over the three diagonal modes. The values of $\kappa$ are chosen to be deep in the SFL phase, $\kappa=0$, as 
well as closer to the QCP, both, in the SFL and QSL phase, i.e. at $\kappa=8$ and 13 , respectively. Several points should be noted. First, in the SFL phase at $\kappa=0$ and 8 , the spectra corroborate a clear Goldstone mode behavior, with a pronounced spectral weight at the $\boldsymbol{\Gamma}$-point. Second, in the QSL phase, the spectral weight may show remnants of this at $j=1$. However, weight is also redistributed into other regions of momentum space. For $j=0.5$, the DSSF in the QSL regime is rather featureless versus momentum, which has to be attributed in part to less statistics for the imaginary time data in this regime of small $j$ and large $\kappa$. Third, introducing ring exchange, the spectrum is shifted to higher energies and is broadened (note the different $y$-scales of the panels). Fourth, Fig. 7 displays an opening of a gap at the $\boldsymbol{\Gamma}$-point as $\kappa$ increases. It is tempting to associate this with the onset of the two-spinon gap for $\kappa>\kappa_{C}$. To substantiate this, larger systems are required to prove a vanishing gap for $\kappa<\kappa_{C}$. This is beyond our analysis.

\section{CONCLUSION}

To summarize, using extensive quantum Monte-Carlo calculations, we have investigated the role of trimerization in the frustrated planar XY spin- $1 / 2$ ferromagnet on the kagome lattice with four-site ring exchange. Among our main findings is that qualitatively, several aspects of the physics of this system already known for the uniform case, are nearly invariant with respect to trimerization, if the exchange parameters are rescaled properly. This pertains to the quantum phase diagram, comprising LRO and $\mathbb{Z}_{2}$ QSL states, the scaling behavior and the universality class, as well as the static structure factor of the model. Regarding the impact of trimerization on the excitations, we find that linear spin waves remain a reasonably valid description in the LRO phase, while spinon continua in the QSL phase may even be intensified in the non-uniform case. Open questions remain, regarding a non-monotonous behavior of the spin stiffness with ring exchange at strong trimerization, which may signal new types of ground states. Regarding actual realizations in optical lattices or local-moment systems, our study shows that, in terms of the boson exchange parameters, trimerization enlarges the QSL regime.

\section{ACKNOWLEDGMENTS}

Work of N.C. and W.B. has been supported in part by the State of Lower Saxony through QUANOMET (project NP-2). Work of W.B. has been supported in part by the DFG through project A02 of SFB 1143 (projectid 247310070). W.B. also acknowledges kind hospitality of the PSM, Dresden. This research was supported in part by the National Science Foundation under Grant No. NSF PHY-1748958.

\section{Appendix A: Spin Stiffness of XY Trimer}

At $j=k=0$, our model simplifies to disconnected $\mathrm{XY}$ trimers on a triangular lattice. In turn, even in this limit the model exhibits a finite extensive spin stiffness, resulting from the trimers. Since this does not imply a collective LRO state, we analyze the stiffness obtained from QMC by subtracting the bare trimer stiffness. The latter can be obtained analytically by introducing a twist $\phi$ along the $x$-direction of the trimer. This affects only the $S^{z}= \pm 1 / 2$ sectors, containing cyclic permutation of $|\uparrow \downarrow \downarrow\rangle$ and $|\downarrow \uparrow \uparrow\rangle$. For the $S^{z}=1 / 2$

$$
H_{\text {trimer }}^{S^{z}=1 / 2}(\phi)=-\frac{J_{\Delta}}{2}\left(\begin{array}{ccc}
|\uparrow \uparrow \downarrow\rangle & |\uparrow \downarrow \uparrow\rangle & |\downarrow \uparrow \uparrow\rangle \\
1 & 0 & 1 \\
1 & e^{-\mathrm{i} \phi} & 0
\end{array}\right)
$$

and identically for $S^{z}=-1 / 2$. Moreover $H_{\text {trimer }}(\phi)$ $|\uparrow \uparrow \uparrow(\downarrow \downarrow \downarrow)\rangle=0|\uparrow \uparrow \uparrow(\downarrow \downarrow \downarrow)\rangle$. The stiffness is obtained from the free energy by $\tilde{\rho}_{S t}(T)=\partial^{2} F(\phi) /\left.\partial \phi^{2}\right|_{\phi=0}$. Straightforward algebra yields

$$
\tilde{\rho}_{S t}(T)=\left[9+\frac{9\left(2\left(e^{\frac{1}{2 T}}+3\right) T+3\right)}{2\left(e^{\frac{3}{2 T}}-1\right) T-3}\right]^{-1} .
$$

Fig. 8 depicts $\tilde{\rho}_{S t}(T) / 4$, where the factor of 4 refers to $L_{\alpha}^{2}$ in Eq. (6) for trimers on a non-interacting kagome lattice with $j=0$, where $L_{\alpha}=2$ because of the $J_{\Delta}$ and (vanishing) $J_{\nabla}$ bonds per spatial direction. The figure also proves that QMC data for $\rho_{S t}(T)$ for a single trimer from Eqns. (5, 6) agrees with Eq. A2.

\section{Appendix B: Linear Spin Wave Theory}

In the SFL phase and for $\kappa=0$, the QMC spectra can be contrasted against magnon excitations obtained from linear spin wave theory (LSWT). For the latter we use the conventional Holstein-Primakoff representation with a quantization axis along $x$ and expanded up to leading order $1 / S$, i.e.

$$
\begin{aligned}
& S_{m}^{x}=S-a_{m}^{\dagger} a_{m} \\
& S_{m}^{y} \approx \frac{\sqrt{2 S}}{2 \mathrm{i}}\left(a_{m}-a_{m}^{\dagger}\right)
\end{aligned}
$$

with Boson operators $a_{m}^{(\dagger)}$. Inserting this into Eq. (2) and after Fourier transformation we get

$$
\mathcal{H}^{(2)}=S \sum_{\mathbf{q}} \Psi_{\mathbf{q}}^{\dagger} \underbrace{\left(\begin{array}{cc}
z \bar{J} \cdot \mathbb{1}-\Gamma(\mathbf{q}) & \Gamma(\mathbf{q}) \\
\Gamma(\mathbf{q}) & z \bar{J} \cdot \mathbb{1}-\Gamma(\mathbf{q})
\end{array}\right)}_{M(\mathbf{q})} \Psi_{\mathbf{q}}
$$

where $z=z_{\Delta_{+}}+z_{\nabla}=2+2=4$ is the coordination number, $\Psi_{\mathbf{q}}=\left(a_{0, \mathbf{q}}^{\dagger} a_{1, \mathbf{q}}^{\dagger} a_{2, \mathbf{q}}^{\dagger} a_{0, \mathbf{q}} a_{1, \mathbf{q}} a_{2, \mathbf{q}}\right)$ is a spinor of creation and annihilation operators, with 0,1 , and 2 referring to kagome basis of the triangular lattice, and $\Gamma(\mathbf{q})$ encodes the hopping matrix elements 


$$
\Gamma(\mathbf{q})=\frac{1}{2}\left(\begin{array}{ccc}
0 & J_{\Delta} e^{\mathrm{i} \mathbf{q} \cdot \mathbf{r}_{1}}+J_{\nabla} e^{-\mathrm{iq} \cdot \mathbf{r}_{1}} & J_{\Delta} e^{\mathrm{iq} \cdot \mathbf{r}_{2}}+J_{\nabla} e^{-\mathrm{iq} \cdot \mathbf{r}_{2}} \\
J_{\Delta} e^{-\mathrm{i} \mathbf{q} \cdot \mathbf{r}_{1}}+J_{\nabla} e^{\mathrm{iq} \cdot \mathbf{r}_{1}} & 0 & J_{\Delta} e^{\mathrm{iq} \cdot\left(\mathbf{r}_{2}-\mathbf{r}_{1}\right)}+J_{\nabla} e^{-\mathrm{iq} \cdot\left(\mathbf{r}_{2}-\mathbf{r}_{1}\right)} \\
J_{\Delta} e^{-\mathrm{iq} \cdot \mathbf{r}_{2}}+J_{\nabla} e^{\mathbf{i q} \cdot \mathbf{r}_{2}} & J_{\Delta} e^{-\mathrm{iq} \cdot\left(\mathbf{r}_{2}-\mathbf{r}_{1}\right)}+J_{\nabla} e^{\mathrm{iq} \cdot\left(\mathbf{r}_{2}-\mathbf{r}_{1}\right)} & 0
\end{array}\right)
$$

The magnon dispersions result from the paraunitary secular equation $\operatorname{det}|S \Lambda \cdot M(\mathbf{q})-\omega \mathbb{1}|=0$, where $\Lambda=\left(\begin{array}{cc}\mathbb{1} & 0 \\ 0 & -\mathbb{1}\end{array}\right)$. We find

$$
\begin{aligned}
\omega_{0,1}^{2} / J_{\Delta}^{2} & =\frac{\bar{\jmath}}{2}\left[6 \bar{\jmath} \mp \sqrt{(3 j-1)^{2}+8[1+j \gamma(\mathbf{q})]}\right] \\
\omega_{2}^{2} / J_{\Delta}^{2} & =\frac{3}{2}(1+j)^{2}=6 \bar{\jmath}^{2}
\end{aligned}
$$

with $\gamma(\mathbf{q})=\cos \left(2 \mathbf{q} \cdot \mathbf{r}_{1}\right)+\cos \left(2 \mathbf{q} \cdot \mathbf{r}_{2}\right)+\cos \left(2 \mathbf{q} \cdot\left(\mathbf{r}_{2}-\mathbf{r}_{1}\right)\right)$. In Eq. (B4), $\omega_{0}$ corresponds to the minus sign on the right hand side and the three branches $\omega_{0}$ and $\omega_{1,2}$ comprise one acoustic and two optical modes. One of the latter is completely flat, i.e. at $\omega_{2}$ the magnons are localized from local interference effects, which are a reoccurring theme for NN-hopping models on the kagome lattice. At the $\mathbf{K}$-point, the gap between the 0 - and 1-mode satisfies
$\Delta_{01}(\mathbf{K}, j) \equiv \omega_{0}(\mathbf{K}, j)-\omega_{1}(\mathbf{K}, j)=\sqrt{3 \bar{\jmath}}(1-\sqrt{j})$. I.e. for $j=1$, the optical and acoustic mode display a touching point at this momentum.

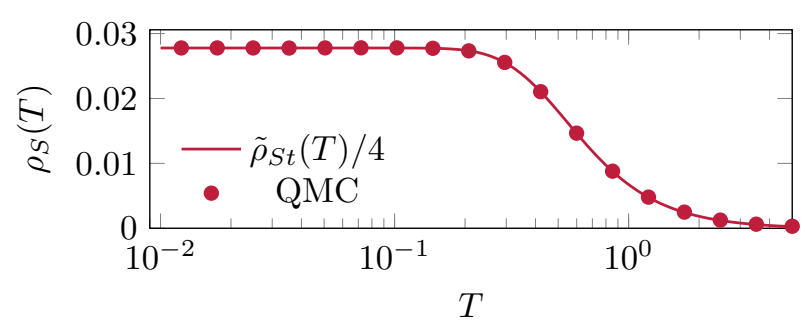

FIG. 8. Comparison of spin stiffness versus $\mathrm{T}$ for single $\mathrm{XY}$ trimer $\tilde{\rho}_{S t}(T) / 4$ from Eq. A2 with QMC trimer result. Errorbars smaller than marker size.
[1] P. W. Anderson, Mater. Res. Bull. 8, 153160 (1973).

[2] P. Fazekas and P. W. Anderson, Phil. Mag. 30, 423 (1974).

[3] G. Misguich and C. Lhuillier, in Frustrated Spin Systems (World Scientific, 2005), pp. 229-306.

[4] L. Balents, Nature 464, 199-208 (2010).

[5] L. Savary and L. Balents, Rep. Prog. Phys. 80, 016502 (2016).

[6] J. Knolle and R. Moessner, Annu. Rev. Condens. Matter Phys. 10, 451 (2019).

[7] X.-G. Wen, Phys. Rev. B 65, 165113 (2002).

[8] P. Henelius and A. W. Sandvik, Phys. Rev. B 62, 1102 (2000).

[9] A. Kitaev, Ann. Phys. (NY) 321, 2 (2006).

[10] J. Nasu, M. Udagawa, and Y. Motome, Phys. Rev. Lett. 113, 197205 (2014).

[11] L. Balents, M. P. A. Fisher, and S. M. Girvin, Phys. Rev. B 65, 224412 (2002).

[12] D. S. Rokhsar and S. A. Kivelson, Phys. Rev. Lett. 61, 2376 (1988).

[13] R. Moessner and S. L. Sondhi, Phys. Rev. Lett. 86, 1881 (2001).

[14] R. Moessner, S. L. Sondhi, and E. Fradkin, Phys. Rev. B 65, 024504 (2001).

[15] D. N. Sheng and L. Balents, Phys. Rev. Lett. 94, 146805 (2005).

[16] S. V. Isakov, Y. B. Kim, and A. Paramekanti, Phys. Rev. Lett. 97, 207204 (2006).

[17] S. V. Isakov, A. Paramekanti, and Y. B. Kim, Phys. Rev. B 76, 224431 (2007).

[18] L. Dang, S. Inglis, and R. G. Melko, Phys. Rev. B 84, 132409 (2011).

[19] S. V. Isakov, M. B. Hastings, and R. G. Melko, Nature
Phys. 7, 772 (2011).

[20] S. V. Isakov, R. G. Melko, and M. B. Hastings, Science 335, 193 (2012).

[21] F. J. Wegner, Journal of Mathematical Physics 12, 2259 (1971).

[22] X. G. Wen, Phys. Rev. B 44, 2664 (1991).

[23] A. Yu. Kitaev, Annals of Phys. 303, 2 (2003).

[24] S. Sachdev, Rep. Prog. Phys. 82, 014001 (2018).

[25] Y.-C. Wang, C. Fang, M. Cheng, Y. Qi, and Z. Y. Meng, arXiv: 1701.01552

[26] J. Becker and S. Wessel, Phys. Rev. Lett. 121, 077202 (2018).

[27] J. Becker and S. Wessel, Phys. Rev. B 100, 241113(R) (2019).

[28] A. V. Chubukov, T. Senthil, and S. Sachdev, Phys. Rev. Lett. 72, 2089 (1994).

[29] A. V. Chubukov, S. Sachdev, and T. Senthil, Nuclear Physics B 426, 601 (1994).

[30] T. Senthil and O. Motrunich, Phys. Rev. B 66, 205104 (2002).

[31] S. V. Isakov, T. Senthil, and Y. B. Kim, Phys. Rev. B 72, 174417 (2005).

[32] H. G. Ballesteros, L. A. Fernández, V. Martín-Mayor, and A. Muñoz Sudupe, Physics Letters B 387, 125 (1996).

[33] P. Calabrese, A. Pelissetto, and E. Vicari, Phys. Rev. E 65, 046115 (2002).

[34] M. Campostrini, M. Hasenbusch, A. Pelissetto, P. Rossi, and E. Vicari, Phys. Rev. B 63, 214503 (2001).

[35] F. Mila, Phys. Rev. Lett. 81, 2356 (1998).

[36] M. Mambrini and F. Mila, Eur. Phys. J. B 17, 651 (2000).

[37] M. E. Zhitomirsky, Phys. Rev. B 71, 214413 (2005).

[38] R. Schaffer, Y. Huh, K. Hwang, and Y. B. Kim, Phys. 
Rev. B 95, 054410 (2017).

[39] C. Repellin, Y.-C. He, and F. Pollmann, Phys. Rev. B 96, 205124 (2017).

[40] Y. Iqbal, D. Poilblanc, R. Thomale, and F. Becca, Phys. Rev. B 97, 115127 (2018).

[41] M. Iqbal, D. Poilblanc, and N. Schuch, Phys. Rev. B 101, 155141 (2020).

[42] L. Santos, M. A. Baranov, J. I. Cirac, H.-U. Everts, H. Fehrmann, and M. Lewenstein, Phys. Rev. Lett. 93, 030601 (2004).

[43] B. Damski, H. Fehrmann, H.-U. Everts, M. Baranov, L. Santos, and M. Lewenstein, Phys. Rev. A 72, 053612 (2005).

[44] P. Windpassinger and K. Sengstock, Rep. Prog. Phys. 76, 086401 (2013).

[45] G.-B. Jo, J. Guzman, C. K. Thomas, P. Hosur, A. Vishwanath, and D. M. Stamper-Kurn, Phys. Rev. Lett. 108, 045305 (2012).

[46] T. H. Barter, T.-H. Leung, M. Okano, M. Block, N. Y. Yao, and D. M. Stamper-Kurn, Phys. Rev. A 101, 011601(R) (2020).

[47] A. W. Sandvik, J. Phys. A 25, 3667 (1992).
[48] A. W. Sandvik, Phys. Rev. B 59, R14157 (1999).

[49] O. F. Syljuåsen and A. W. Sandvik, Phys. Rev. E 66, 046701 (2002).

[50] R. G. Melko and A. W. Sandvik, Phys. Rev. E 72, 026702 (2005).

[51] A. Cuccoli, T. Roscilde, V. Tognetti, R. Vaia, and P. Verrucchi, Phys. Rev. B 67, 104414 (2003).

[52] A. W. Sandvik, Phys. Rev. B 56, 11678 (1997).

[53] E. L. Pollock and D. M. Ceperley, Phys. Rev. B 36, 8343 (1987).

[54] K. Harada and N. Kawashima, J. Phys. Soc. Jpn. 67, 2768 (1998).

[55] J. Skilling and R. K. Bryan, Mon. Not. R. Astron. Soc. 211, 111 (1984).

[56] R. Bryan, Eur. Biophys. J. 18, 165174 (1990).

[57] M. Jarrell and J. Gubernatis, Phys. Rep. 269, 133 (1996).

[58] M. P. A. Fisher, P. B. Weichman, G. Grinstein, and D. S. Fisher, Phys. Rev. B 40, 546 (1989).

[59] In [18] $J$ is rescaled by a factor of 2 , as compared to this work, resulting in $(K / J)_{c} \approx 21.8$. 